\title{
LA AUTOEXPOSICIÓN Y PREVENCIÓN DE RESPUESTA EN UN CASO DE TRASTORNO OBSESIVO-COMPULSIVO CON RITUALES DE COMPROBACIÓN
}

\author{
Cristina Robert Flors \\ Unidad de Salud Mental de Vila-Real (Castellón) \\ Universidad Jaume I (Castellón)
}

\begin{abstract}
RESUMEN
El presente trabajo se enmarca dentro del esfuerzo observado en los últimos años en el ámbito de la psicologia clínica por disminuir el coste de los tratamientos psicológicos. Se presenta un caso de trastorno obsesivo-compulsivo con rituales de comprobación, tratado en un centro de asistencia público con un programa de tratamiento de exposición y prevención de respuesta. La aplicación del tratamiento fue llevada a cabo por completo por el paciente, limitándose el papel del psicólogo a la explicación de la técnica, la supervisión de su aplicación y refuerzo de los logros. EI tratamiento se realizó en ocho sesiones de unos 30-45 minutos de duración, a lo largo de cuatro meses. Se ofrecen resultados obtenidos al finalizar la terapia, a los ocho meses y al año de seguimiento.
\end{abstract}

Palabras clave: Trastorno obsesivo-compulsivo; Tratamiento psicológico,; Autoexposición

\begin{abstract}
During the last years, a major effort has been developed to reduce the cost of psychological treatments in the clinical psychology field. This study is framed in this context. An obsessive-compulsive disorder with checking behavior is presented, and resolved with an exposure and response prevention treatment program. This program was carried out by the patient, where the psychologist role was reduced to teaching the technique to the patient, supervising its application, an enhancing the success. The treatment was carried out in eight sessions of 30-45 minutes during four months. Results at the end of therapy and after eight months and a year.
\end{abstract}

Key words: Obsessive-compulsive disorder; Psychological Treatment; Autoexposure. 


\section{INTRODUCCIÓN}

Los trastornos de ansiedad presentan una alta prevalencia entre los pacientes que acuden a las consultas de atención primaria y entre aquellos que acuden a recibir ayuda especializada (Marks, 1987). Entre éstos, el trastorno obsesivo-compulsivo es uno de los que causan un mayor grado de malestar e incapacidad en aquellas personas que lo padecen. Es a partir de mediados de los años sesenta (Meyer, 1966) y, sobre todo, a partir de la década de los setenta, cuando, con el surgimiento de las técnicas de exposición y prevención de respuesta, el pronóstico para estos pacientes parece mejorar obstensiblemente (Hodgson y Rachman, 1977; Marks, 1975). En la actualidad, podemos decir que éste es el tratamiento de elección para la mayoria de estos pacientes (Cruzado, 1993; Greist, 1992), ya que éstas técnicas han demostrado ser eficaces a largo plazo para el tratamiento de este trastorno (Marks, 1987; Robert y Botella, 1990; Marks y Sullivan, 1992; De Silva y Rachman, 1995). Es por esto que, en el momento actual, el interés de investigadores y clínicos está dirigido, no tanto hacia la consecución de técnicas de tratamiento útiles, como a intentar disminuir el coste de estos tratamientos, manteniendo su eficacia.

El coste de las terapias psicológicas se debe principalmente a la necesidad de presencia activa por parte del terapeuta. Por esta razón, este tiempo de dedicación del terapeuta por paciente se ha intentado reducir de diferentes modos: utilizando un formato de grupo (Méndez, Olivares y Macià, 1993), disminuyendo el número de sesiones de terapia, utilizando como coterapeuta a personal auxiliar (Ruipérez, Robert y Botella, 1994), proporcionando materiales de auto-ayuda (Pruitt, Mille y Smith, 1989), y fomentando la autoexposición, con o sin ayuda de coterapeuta.

El presente trabajo se enmarca dentro de este esfuerzo por disminuir el coste de los tratamientos psicológicos, ofreciendo un programa de tratamiento de exposición y prevención de respuesta, sin tratamiento farmacológico coadyuvante, que fue llevado a cabo por completo por el paciente, limitándose el papel del psicólogo a la explicación de la técnica, la supervisión de su aplicación y el refuerzo de los logros.

\section{MÉTODO}

\subsection{Descripción del caso}

El paciente es un hombre de 39 años, de clase social media, casado hace trece años y con relación de pareja estable. Tiene una hija de 11 años. Respecto a su familia de origen, es el mayor de tres hermanos, y es el único varón. Terminó los estudios primarios y trabaja como esmaltador en una empresa de cerámica. Vive en un pueblo de 18.000 habitantes. Acude en agosto de 1995 a la Unidad de Salud Mental remitido por su médico de cabecera por la existencia de diversos rituales de verificación.

Se comporta de manera educada y muy formal durante toda la entrevista. Presenta un aspecto saludable y fuerte, pulcro y aseado Su discurso es coherente. En ocasiones presenta dificultades para explicar su problema y necesita tiempo, ya que es meticuloso con sus explicaciones. Aparenta encontrarse en tensión todo el rato, suda y gesticula con frecuencia. Al preguntarle si se encuentra nervioso, contesta negativamente. Viene acompañado por su mujer, de 32 años, que presenta un aspecto más relajado que el marido. Esta se muestra atenta y comprensiva durante la entrevista. La interacción entre los dos es correcta.

El paciente se define a sí mismo como una persona educada, respetuosa, responsable y perfeccionista. Parece mantener sus emociones bajo un estricto control. Tiene una alta autoestima (se auto-califica como "buena persona, honrado, trabajador, etc"). Mantiene buenas relaciones familiares. También presenta una buena adaptación laboral, pues le gusta su trabajo y valora positivamente las relaciones con los compañeros.

Relata que el embarazo de su madre, el parto y su propio desarrollo evolutivo fueron normales. Verbaliza una buena relación familiar y adaptación escolar. No presenta problemas psicológicos anteriores. Operado de hernia inguinal a los 25 años. Rechazó los puntos de la primera operación diez años después, por lo que tuvo que ser nuevamente operado. Dice que pasó mucho miedo. Su estado de salud actual es bueno. No presenta problemas de sueño ni alimentación. 


\subsection{Motivo de consulta}

En el momento actual presenta rituales de comprobación que aparecen en las situaciones de desplazamientos, sea conduciendo, en bicicleta o andando, principalmente, cuando adelanta a alguien o entra en una rotonda de giro, y en respuesta a obsesiones de duda sobre la posibilidad de haber hecho algo mal. El nivel de perturbación es moderado, pues la evitación reduce el malestar y los rituales no implican actividades vitales para el sujeto.

Indica que cuando entra en una rotonda conduciendo, piensa que "ha podido hacer algo que no está bien". Da la vuelta y hace de nuevo el recorrido para comprobar que todo está en orden. Estas verificaciones las suele realizar varias veces seguidas, hasta que se queda tranquilo. En ocasiones es tan alta la ansiedad que ha necesitado llegar a casa, recoger a su mujer y volver al mismo sitio para asegurarse de que no ha pasado nada malo.

Algo parecido le ocurre cuando va en bicicleta, cuando adelanta a alguien en coche $e$ incluso cuando va andando. Piensa que ha podido hacer algo incorrecto, por lo que se detiene varias veces para comprobarlo. En el momento actual, y como un modo de evitar el malestar, no coge el coche desde hace algunos meses.

Hace unos veinte años dió un golpe con el coche a una moto, sin mayores consecuencias, pero él cree que pudiera tener relación con sus problemas actuales. Los rituales de comprobación se iniciaron coincidiendo con la compra de un coche nuevo. Al principio sólo le ocurria en las rotondas, pero, de modo gradual, se ha ido generalizando a más situaciones y ha ido aumentando el número de comprobaciones que debe realizar.

No sabe a qué atribuir su problema. Dice que "tiene manias" y las encuentra absurdas, pero no puede resistirse a ellas. Nunca ha recibido tratamiento farmacológico ni psicológico por éste $u$ otros problemas.

\section{EVALUACIÓN}

Se llevó a cabo a lo largo de dos sesiones, y se utilizaron los siguientes instrumentos:

- Entrevista clínica: proporcionó la información necesaria para desarrollar la historia clinica y evaluar las conductas objeto del tratamiento.
- Inventario de Depresión de Beck (1961) (versión 21 items).

- Inventario de Autoevaluación de la Ansiedad Estado-Rasgo (STAI) (Spielberger, Gorush y Lushene, 1970), para valorar el nivel de ansiedad en general (ansiedad-estado).

- Escala de Adaptación de Echeburúa y Corral (1987)

Para evaluar el trastorno obsesivo-compulsivo se utilizaron los siguientes instrumentos:

- Compulsive Activity Checklist (CAC) (Philpott, 1975).

- Inventario de Obsesión-Compulsión del Maudsley (Hodgson y Rachman, 1977).

- Escala ET (adaptación de la escala S-1 de Marks, 1975): se establecieron conjuntamente entre paciente y terapeuta las conductas objetivo (situaciones o actividades que evita). La escala permite valorar en cada sesión el grado de evitación y temor para cada una de ellas (de $0=$ nada a $10=$ muchísimo):

- Registro de conductas de comprobación, durante un período de 10 días, de línea-base, antes de iniciar el tratamiento (Anexo 1).

\subsection{Diagnóstico y pronóstico}

Según el sistema de clasificación diagnóstica del DSM-IV (American Psychiatric Association, 1994):

Eje 1: F 42.8 Trastorno Obsesivo-Compulsivo [300.3]

Eje 2: Z $\quad$ 03.2 No hay diagnóstico, caracteristicas de personalidad obsesivo-compulsiva

[V71.09]

Eje 3: Ninguno.

Eje 4: Ninguno.

Eje 5: $E E A G=60$

El pronóstico es favorable, debido a la alta motivación del paciente y de su pareja hacia la terapia, el alto valor que otorga a la realización correcta de las tareas, su nivel intelectual, la no existencia de otros problemas concomitantes $y$ el hecho de que nunca haya intentado una terapia de exposición y prevención de respuesta.

\subsection{Psicopatograma}

No presenta alteraciones del lenguaje, estado de conciencia, curso del pensamiento, 
memoria, percepción, orientación, inteligencia, ni juicio y creencias. Respecto al contenido del pensamiento, se evidencia la existencia de pensamientos obsesivos. En cuanto al nivel de ansiedad, presenta una ansiedad difusa de la que no es del todo consciente. Reconoce estar enfermo y necesitar ayuda, y es capaz de informar con exactitud sobre sus sintomas.

\subsection{Análisis funcional}

Problema 1: conductas de comprobacion en los desplazamientos.

- Situaciones desencadenantes:

al conducir, entrar en una rotonda, adelantar a peatones, ciclistas o motos.

al pasear con la bicicleta por la montaña.

cuando va andando por la calle, sobre todo, si se cruza con otra persona.

- Respuestas cognitivas: piensa que ha podido hacer algo que no está bien, haberle hecho daño a alguien. Sabe que no ha hecho nada malo, pero necesita cerciorarse. Si no puede realizar los rituales, está pensando en ello durante 10-15 minutos.

- Respuestas fisiológicas: En el registro de linea-base se observa que el paciente realizó conductas de comprobación en todas las ocasiones en que se enfrentó a las situaciones desencadenantes, con un nivel de ansiedad leve (valoración media de 3 , en una escala de valoración de 0-10). El paciente informa que cuando no puede realizar los rituales, presenta una ansiedad más elevada (nivel de 6), que él define como intranquilidad, pero que empieza a disminuir a los 10-15 minutos.

- Respuestas motoras: múltiples y repetidas conductas de comprobación: mirar hacia atrás, repetir el recorrido, mirar por el espejo retrovisor. En el registro de línea base, estas conductas aparecen a diario: mira hacia atrás con una media de unas diez veces al día, de 3 a 10 veces cada vez, repite el recorrido con una media de una repetición, una vez al dia. En el último mes, presenta conductas de evitación: no coge el coche. Se le pregunta qué ocurria antes de evitar la conducción y el paciente indica que las repeticiones del recorrido con el coche, al volver del trabajo, solian tener una duración de unas dos horas.

- Consecuencias: disminución de la ansiedad después de varias repeticiones.
Problema 2: conductas de comprobacion en casa.

- Situaciones desencadenantes: el momento de acostarse.

- Respuestas cognitivas: "no sé si estarán bien cerradas".

- Respuestas fisiológicas: no reconoce activación vegetativa.

- Respuestas motoras: comprueba varias veces el gas, la televisión, la puerta de la calle, con una duración media de 10 minutos diarios.

- Consecuencias: se acuesta tranquilo.

En todas las situaciones anteriores, se siente "liberado" de sus dudas y no necesita realizar la comprobación si "traspasa la responsabilidad" a otra persona (si su mujer comprueba el gas, conduce otra persona, etc). También disminuye su ansiedad cuando conduce en caravana (piensa "de haber hecho algo malo, me avisarian").

Problema 3: otros problemas relacionados.

Perfeccionista y preocupado por los detalles. Sus compañeros le dicen que es meticuloso y ordenado en el trabajo. Siente con frecuencia que no ha terminado bien determinadas tareas, pero no realiza conductas de comprobación en el trabajo. Estas preocupaciones le duran minutos y no interfieren la marcha de su trabajo. Tiene miedo al fracaso.

Preocupaciones ocasionales generadoras de ansiedad sobre si le podria pasar algo a su hija, si podría desarrollar alguna enfermedad... Principalmente cuando lee o escucha alguna noticia sobre enfermedad o accidentes. Pero informa de que le duran minutos y que es capaz de "desengancharse".

Fobia a la sangre. Suele marearse, aunque no ha perdido el conocimiento. Presenta conductas de evitación solapadas: en las revisiones médicas de la empresa, solicita ser el primero en la extracción, para evitar la ansiedad anticipatoria.

\subsection{Interferencia del problema en la vida del paciente}

Para valorar este punto se le pasó el cuestionario de Echeburúa y Corral (1987), observándose lo siguiente:

- Area social: leve.

- Area laboral: moderado. Evita coger el coche, por lo que debe ir a trabajar en autobús 
y debe pedir a un compañero que lo acompañe en los desplazamientos. Si se ve obligado a conducir, tarda el doble de lo normal en volver a casa, debido a las conductas de comprobación.

- Area familiar: moderado. Aunque su mujer le dice que "son tonterias" y que no los realice $y$ en ocasiones debe acompañarlo a realizar los rituales de comprobación, el paciente manifiesta que éstos "no interfieren de modo significativo en sus relaciones familiares".

- Area personal: moderado. Tiende a evitar actividades con las que antes disfrutaba, como salir por la montaña con bicicleta. En los desplazamientos se siente inseguro y necesita más tiempo del normal.

\section{TRATAMIENTO}

El paciente indicó que no deseaba recibir tratamiento farmacológico, "prefiere antes intentarlo sin medicamentos". La terapia psicológica se inició a finales de Septiembre de 1995. El tratamiento de elección consistió en la exposición sistemática a aquellas situaciones que desencadenaban las conductas de comprobación, junto con la prevención de tales respuestas.

El tratamiento tuvo una duración total de 8 sesiones, a lo largo de cuatro meses. Las sesiones de terapia se llevaron a cabo cada 10-15 días y tuvieron una duración aproximada de 30-45 minutos (excepto la primera sesión, que duró una hora y cuarto). El retest se llevó a cabo 15 semanas después de iniciado el tratamiento. El primer seguimiento tuvo lugar a los ocho meses de la finalización del mismo, $y$ el segundo seguimiento al año.

La primera sesión de tratamiento se dedicó a explicar al paciente y a su esposa los resultados de la evaluación, asi como los fundamentos de la terapia. El contenido de la sesión fue el siguiente:

1. Explicación de los resultados de la evaluación, especialmente, los datos obtenidos en el análisis funcional.

2. Explicación del papel adaptativo de la ansiedad y de las formas de manifestarse (los tres sistemas de respuesta).

3. Explicación del papel de la evitación $y$ los rituales en el mantenimiento del problema.
4. Explicación de las técnicas de exposición y prevención de respuesta.

5. Realización en colaboración con el paciente y su esposa de la jerarquía de situaciones de exposición.

6. Explicación a la esposa del paciente de su papel como coterapeuta: reforzar los logros, ayudarle a planificar las sesiones de exposición, no reforzar las dudas (no proporcionar al paciente seguridad sobre la correcta realización de una conducta).

7. Planificación de las tareas hasta la próxima sesión.

Tras cada una de las explicaciones se pidió a cada uno de los cónyuges su opinión sobre lo expuesto, en qué medida era coherente con su experiencia y se les solicitó un breve resumen, para aseguramos de que lo habian entendido con claridad. Asimismo, se dedicó la sesión a fomentar la motivación hacia el tratamiento y a recalcar la importancia de una implicación activa en el mismo.

Se negociaron con el paciente los objetivos de terapia y éstos fueron los siguientes:

1. Girar conduciendo solo en una rotonda y no comprobar por el retrovisor ni volver hacia atrás.

2. Ir andando (sin adelantar a nadie) y no mirar atrás.

3. Adelantar andando a alguien y no mirar hacia atrás.

4. Adelantar en coche una bicicleta o una moto y no mirar atrás.

5. Comprobar una sola vez el gas y la puerta.

6. No mirar atrás ni retroceder en bicicleta.

Tal y como se observa en la tabla $n^{0} 1$, se obtuvieron dos jerarqulas de situaciones para iniciar la exposición, una para cada una de las situaciones-problema.

Se pactaron entre el terapeuta y ambos cónyuges las tareas de exposición. Las instrucciones fueron: realizar una exposición diaria de un hora y media de duración (mínimo una hora), empezando por el objetivo 1 de la primera jerarquía. Cuando la ansiedad máxima fuera inferior a 3, continuar con el siguiente objetivo. El paciente debia anotar la ansiedad que sentia en las hojas de registro de exposición (Anexo 2), cada 10 minutos o cuando notara un cambio en el nivel de ansiedad, utilizando una escala USA (Unidades 
Subjetivas de Ansiedad) de 0 (nada de ansiedad) a 10 (muchísima ansiedad). No podia terminar la exposición antes de observar una disminución (aunque fuera mínima) en su nivel de ansiedad. El resto del dia, no debia de comprobar en ninguna de las situaciones donde lo hacia en el pasado (prevención de respuesta).

Además, el paciente se comprometió a practicar a diario la prevención de respuesta en las conductas de comprobación en casa.

Se les proporcionaron una serie de instrucciones escritas que resumian la información facilitada en terapia (Anexo 3), y se les dió posibilidad de llamar al terapeuta por teléfono si surgian dificultades. También se estableció la cita para la siguiente sesión.

Las siguientes sesiones se dedicaron a revisar las tareas, reforzar el progreso, comentar las dificultades y programar las siguientes prácticas de exposición y prevención de respuesta. En cada sesión se pedia al paciente que valorara el nivel actual de evitación y temor de los objetivos iniciales de terapia.

El sujeto realizó las tareas de exposición con una frecuencia casi diaria. En total, realizó un total de 65 sesiones de autoexposición, repartidas a lo largo de 59 dias (algunos dias realizo dos sesiones).
Empezó la primera sesión de exposición con la conducta "Andar por el pueblo en compañia de mi mujer" (la valoración de los niveles subjetivos de ansiedad en esta primera sesión fueron: ansiedad inicial $=3$; máxi$m a=3$ y al acabar= 3). Este objetivo lo practicó durante las 6 primeras sesiones de exposición. En las siguientes tres sesiones, comenzó a quedar con su mujer cada 15-30 minutos (ansiedad máxima $=1$ ). Durante doce sesiones más, el sujeto anduvo solo una hora diaria por el pueblo, sin realizar conductas de comprobación (ansiedad máxima= 1).

En la sesión 18 de exposición, abordó el objetivo de "Conducir el coche por el pueblo". Condujo acompañado por su mujer en la sesión $18,19,28,29$ y 30 de exposición, con una ansiedad máxima de 2. De la sesión 37 a la 49 realizó de modo diario una sesión de exposición, conduciendo sólo por el pueblo durante una hora, con una ansiedad máxima de 1.

En la sesión 20 comienza a exponerse al objetivo "Conducir por carretera, entrando en rotondas de giro, sin realizar conductas de comprobación". Este objetivo lo practico durante trece sesiones, con una ansiedad máxima de 1. A partir de la sesión 38 inicia la exposición a este objetivo sin compañia. El primer dia, realiza una vuelta de repetición en

Tabla 1.- Jerarquías de exposición a las conductas-objetivo

Problema 1: conductas de comprobación on los desplazamientos
1) Andar por la calle acompañado de su mujer, sin mirar hacia atrás.
2) Andar por la calle solo, sin mirar hacia atrás, quedando con su mujer cada 15 minutos.
3) Lo mismo, pero quedando con su mujer cada media hora.
4) Lo mismo, pero su mujer le espera en casa.
5) Lo mismo, pero su mujer no está en casa mientras dura la exposición.
6) Conducir en coche por el pueblo, acompañado. Adelantar y no comprobar.
7) Lo mismo, pero conduciendo solo.
8) Conducir en bicicleta y no girarse para comprobar.
9) Conducir por carretera sin comprobar. Acompañado.
10) Lo mismo pero solo.
11) Girar en rotondas sin retroceder ni mirar para comprobar. Acompañado.
12) Lo mismo pero solo.

\section{Problema 2: conductas de comprobacion en casa}

1) Cerrar el gas antes de acostarse y no volver para comprobarlo.

2) Echar los cerrojos de la puerta antes de acostarse y no volver para comprobarlo. 
una de las rotondas porque "con el sol no habia visto bien y podia haber ocurrido algo". Al llegar a casa, aborda esta dificultad con su mujer, y no vuelve a repetir ningún recorrido. Si se observan, en las primeras sesiones algunas conductas aisladas (no más de dos veces seguidas) de comprobación por el espejo retrovisor. La ansiedad máxima en las once sesiones de exposición a este objetivo es de uno.

Las sesiones 29 y 32 las dedicó al objetivo de "circular en bicicleta sin realizar conductas de comprobación", con una ansiedad máxima de 0 .

La prevención de respuesta en las conductas de "comprobación del gas y la puerta" se llevó a cabo desde el primer dia.

Una vez llevadas a cabo las sesiones de exposición a cada una de las conductas-objetivo, el sujeto las incorporaba a su vida cotidiana. En ningún momento la ansiedad durante las exposiciones fue alta (ansiedad máxima= 3), a pesar de las valoraciones que el sujeto dió a algunas de las conductas-objetivo antes de iniciar el tratamiento. Aunque el paciente tuvo dificultades en alguna ocasión para aplicar la prevención de respuesta (girar la cabeza alguna vez andando, o mirar por el espejo retrovisor), en ninguna de las ocasiones repitió estas conductas más de una vez.

\section{RESULTADOS}

En la valoración de las conductas objetivo (gráfico 1) se aprecia una disminución continuada y progresiva de la ansiedad y de la evitación a lo largo de las sesiones de terapia. Sólo se observa una excepción en este patrón en la segunda sesión, cuando el paciente acaba de iniciar la exposición. Aqui el paciente informa de un incremento en la valoración subjetiva de la ansiedad en las conductasobjetivo números $1,3,4$ y 5 . No es dificil entender este incremento si tenemos en cuenta que hasta ese momento el paciente habia evitado esas conductas.

Cabe subrayar que la ansiedad y la evitación desaparecen totalmente al finalizar la terapia y que esta importante mejoria se mantiene en los seguimientos.

Por otra parte, tal y como se observa en la tabla número 2, se aprecia una disminución en la puntuación de todas las variables clinicas relacionadas con el trastorno obsesivocompulsivo propiamente dicho. Antes de iniciar la terapia, el paciente obtuvo una puntuación total en el CAC (Philpott, 1975) de 7: puntuó con un $2^{1}$ el item "volver sobre sus propios pasos" y con un $1^{2}$ en los items "tocar las manecillas de las puertas", "abrir y cerrar puertas y ventanas", "andar por la calle", "tirar cosas" y "conducir el coche". Sin embargo, al finalizar la terapia y en los seguimientos se observaba una puntuación de cero (1 en el seguimiento realizado a los ocho meses de finalización de la terapia). En el Inventario de Obsesión-Compulsión del Maudsley (Hodgson y Rachman, 1977), puntuó en la mayoría de items que valoran el componente de "comprobación". Antes de la terapia se observa una puntuación de 10 , que disminuye a la mitad cuando finaliza la terapia y continua observándose una mejoría progresiva en los seguimientos.

Tabla 2.- Puntuaciones en los distintos cuestionarios antes de iniciar la terapia, al finalizar ésta y en los distintos seguimientos

\begin{tabular}{|l|cccc|}
\hline & $\begin{array}{l}\text { Pre- } \\
\text { Terapia }\end{array}$ & Alta & $1^{\circ}$ Seg. & $2^{\circ}$ Seg. \\
\hline C.A.C. & 7 & 0 & 1 & 0 \\
I.O.C.M. & 10 & 5 & 4 & 2 \\
BDI & 5 & 1 & 1 & 0 \\
STAI-R & 8 & 4 & 3 & 9 \\
& & & & \\
\hline
\end{tabular}

Por lo que respecta a la severidad de la depresión y ansiedad-rasgo, el sujeto partía de un nivel preterapia no clínico (no depresión en el BDI y un centil de 5 en la ansiedadrasgo). Estos niveles se mantienen tanto al finalizar la terapia como en los seguimientos, apreciándose una progresiva disminución en ambos a medida que pasa el tiempo, excepto en el último seguimiento, donde se observa un aumento en el nivel de ansiedad-rasgo, aunque ésta sigue siendo baja (centil 11).

Es de señalar que en el último seguimiento, al año de finalización de la terapia, el paciente relata que en una ocasión (un mes 
90

C. ROBERT

Conducta-objetivo $n^{\circ} 1$ :

"Conduciendo, entrar en la rotonda sin comprobar".

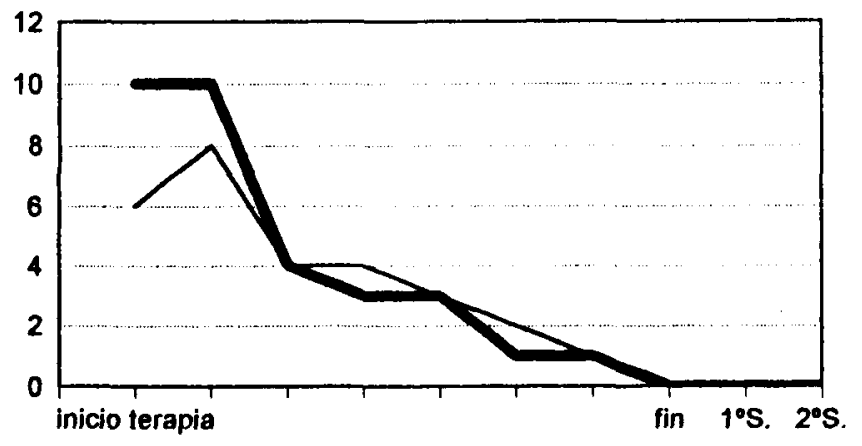

Conducta-objetivo n²: "Ir andando y no mirar atras".

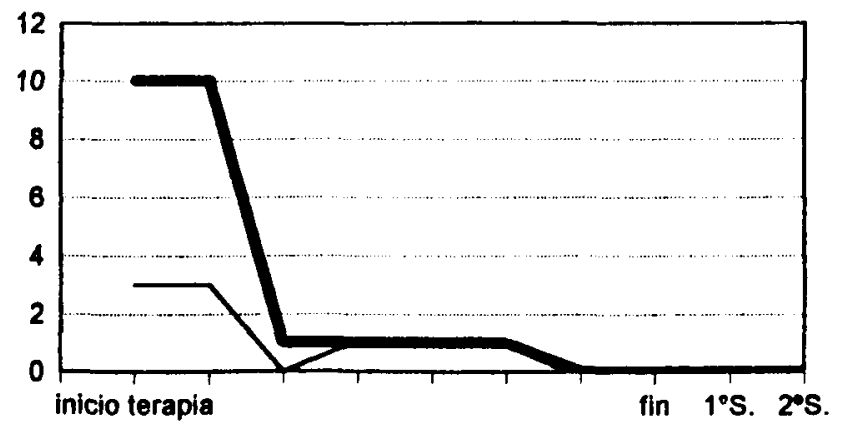

Conducta-objetivo $n^{\circ}$ 3: "Adelantar a alguien y no mirar atras".

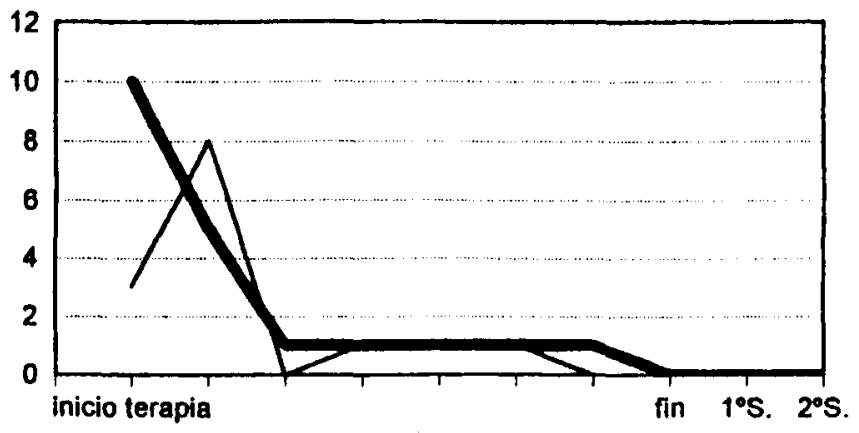

- Evitacion - Temor

Gráfico 1 (a).- Valoración de la evitación y temor de las conductas-objetivo 
Conducta-objetivo $n^{\circ} 4:$

"Conduciendo, adelantar una bici o moto sin comprobar".

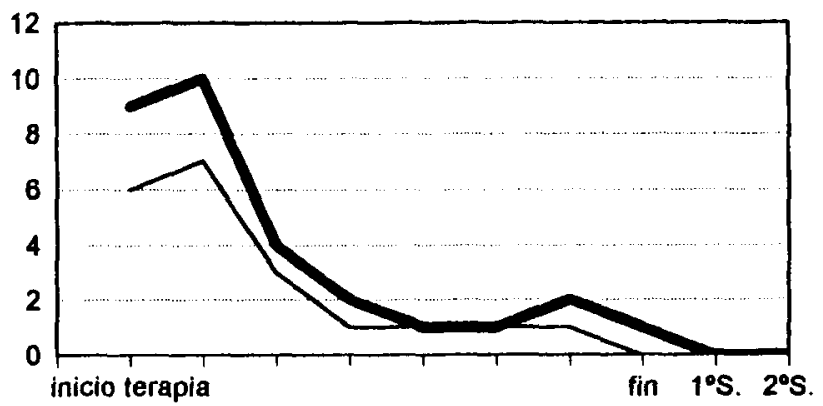

\section{Conducta-objetivo n 5: "No comprobar el gas ni la puerta".}

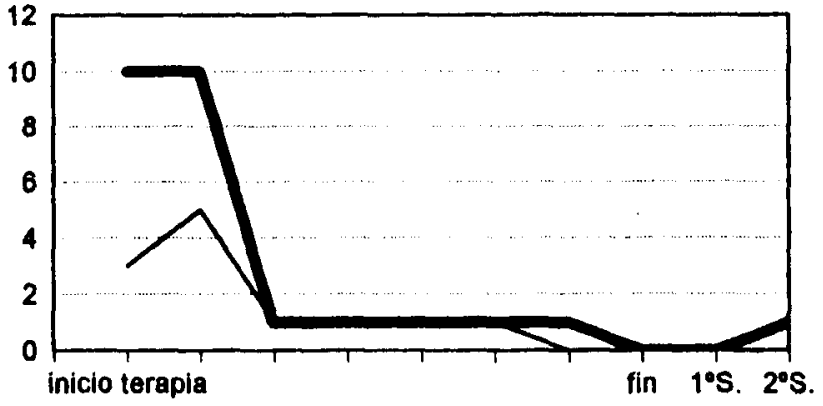

Conducta-objetivo $n^{\circ} 6$ :

"Andando en bicicleta, no mirar atras ni retroceder para comprobar"

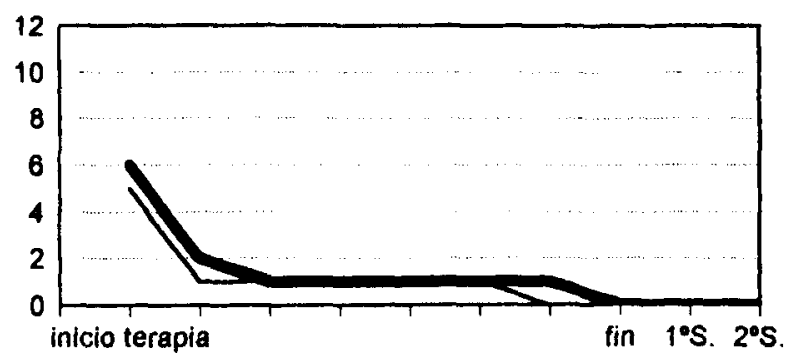

-Evitación - Temor 
antes de acudir) realizó una conducta de comprobación. Coincidió con una época de mayor volumen de trabajo. Al entrar en una rotonda, se dió cuenta de que habia entrado abstraido, pensó "no estoy prestando toda la atención que debo" y dió otra vuelta, para comprobar que "no habia hecho nada mal". Al llegar a casa lo habló con su mujer y decidió durante unos dias hacer sesiones de exposición (coger el coche por rotondas) y prevención de respuesta. El terapeuta reforzó la autoaplicación de las estrategias de prevención de recaidas.

\section{CONCLUSIONES}

Como se ha ido poniendo ya de manifiesto en otros trabajos (Ruipérez et al., 1994), los resultados del presente trabajo permiten ser optimistas sobre la posibilidad de ofrecer tratamiento psicológico eficaz para los trastornos de ansiedad a bajo coste: es posible conseguir una reducción importante en las conductas de evitación y las compulsiones con una participación minima por parte del terapeuta.

En este caso observamos como ha sido posible eliminar estas conductas, que estaban causando un marcado malestar tanto al paciente como a su familia, mediante técnicas de exposición y prevención de respuesta autoaplicadas, reduciéndose el tiempo real de dedicación del terapeuta a ocho sesiones. Además, los resultados se mantienen a lo largo del tiempo (un año de seguimiento), habiendo adquirido el paciente la capacidad de hacer frente por si solo a posibles recaidas, siendo capaz de planificar sesiones de exposición y prevención de respuesta sin la ayuda del terapeuta cuando esto fue necesario. De este modo, parece posible ofrecer ayuda a bajo coste, desde centros públicos, para un problema altamente incapacitante, como es el trastorno obsesivo-compulsivo.

No queremos pecar de optimistas sobre este resultado, ya que se trata, obviamente, de un estudio de caso, que, además, no muestra niveles clinicos de depresión y ansiedad pre-tratamiento. Es necesaria la realización de estudios clínicos controlados antes de que podamos generalizar estos resultados al conjunto de pacientes que presentan el mismo diagnóstico.

\section{REFERENCIAS BIBLIOGRÁFICAS}

American Psychiatric Association (1994): Diagnostic and Statistical Manual of Mental Disorders: DSMIV. (versión en castellano, MASSON, S.A., Barcelona, 1995).

Beck, A. (1961): An inventory for measurement of depression. Archives of General Psychiatry, A, 561-571.

Cruzado, J.A. (1993): Tratamiento comportamental del trastorno obsesivo-compulsivo. Madrid, Fundación Universidad-Empresa.

De Silva, P. y Rachman, S. (1995): Trastorno obsesivo-compulsivo: los hechos. Bilbao, DDB.

Echeburua, E. y Corral, P. (1987): Escala de Adaptación. Manuscrito no publicado.

Greist, J.H. (1992): An integrated approach to treatment of obsessive-compulsive disorder. Journal of Clinical Psychiatry, 53, 38-41.

Hodgson, R.J. y Rachman, S.J. (1977): Obsessional-compulsive complaints. Behaviour Research and Therapy, 15, 389-395.

Marks, I. (1975): Behavioral treatments of phobic and obsessive-compulsive disorders: A critical appraisal. En R. Hersen y cols. (Eds.): Progress in behavior modification. N.Y., Academic Press.

Marks, 1. (1987): Fears, phobias, and rituals. Oxford, Oxford University Press.

Marks, I. y Sullivan, G. (1992): Psicofármacos y tratamientos psicológicos en la agorafobia/pánico y en los trastornos obsesivo-compulsivos. En E. Echeburúa (Ed.): Avances en el tratamiento psicológico de los trastornos de ansiedad. Madrid, Ediciones Pirámide, S.A.

Mathews, A.M.; Gelder, M.G. y Johston, D.W. (1981): Agoraphobia: Nature and treatment. New York. The Guildford Press.

Méndez, F.X.; Olivares, J. y Maciá, D. (1993): Modificación de conducta y programas aplicados a grupos. En D. Macià, F.X. Méndez y J. Olivares (Eds.): Intervención psicologica: Programas aplicados de tratamiento. Madrid, Ediciones Pirámide, S.A.

Meyer, V. (1966): Modification of expectations in cases with obsessional rituals. Behaviour Research and Therapy, 4, 273-280.

Philpott, R. (1975): Recent advances in the behavioral measurement of obsessional illness: Difficulties common to these and other instruments. Scottish Medical Journal, 20, 3340.

Pruitt, S.D.; Miller, W.R. y Smith, J.E. (1989): Outpatient behavioral treatment of severe obsessive-compulsive disorder: Using paraprofessional resources. Journal of Anxiety Disorders, 3, 179-186.

Robert, C. y Botella, C. (1990): Una revisión de los tratamientos psicológicos para los trastornos 
obsesivo-compulsivos. II Congreso Oficial de Psicólogos. Psicologia y Salud: Psicología Clinica. Valencia, del 15 al 20 de Abril.

Ruipérez, M.A.; Robert, C. y Botella, C. (1994): La "Exposición sin terapeuta" en el tratamiento de un trastorno obsesivo-compulsivo. Análisis y Modificación de Conducta, 20, 793-816.
Salkovskis, P.M. y Kirk, J. (1989): Obsessional disorders. En K. Hawton, P.M. Salkovskis, J. Kirk y D.M. Clark (Eds.): Cognitive behaviour therapy for psychiatric problems. A practical guide. Oxford, Oxford University Press.

Spielberger, C.D.; Gorush, R.L. y Lushene, R.D. (1970): Manual for the state-trait inventory. Palo Alto, California. Consulting Psychologist Press.

\section{NOTAS}

1 "Necesita tres veces más de tiempo que los demás, o debe repetirlo tres o más veces o, generalmente, lo evita".

2 "Necesita, por lo menos, el doble de tiempo que los demás o necesita repetirlo o tiende a evitarlo". 
C. ROBERT

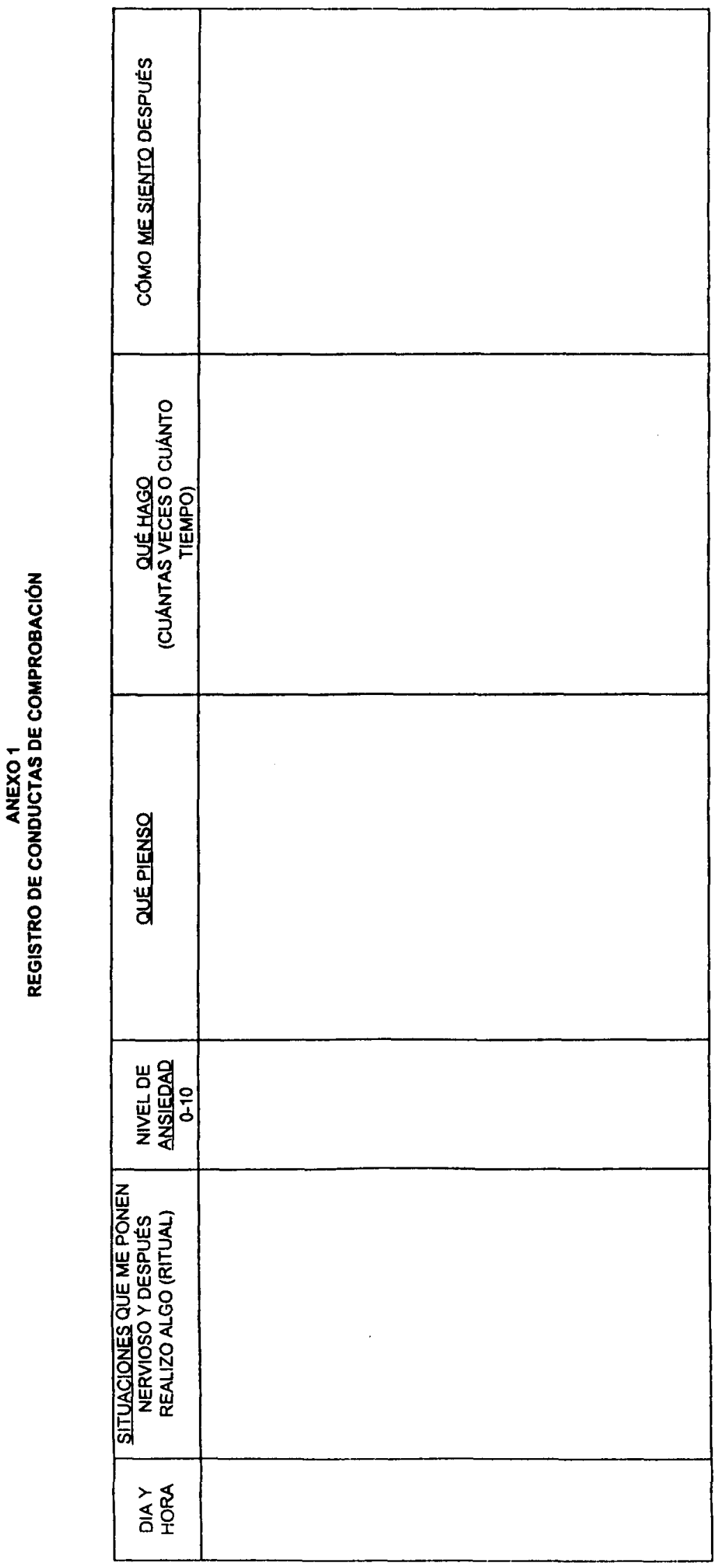




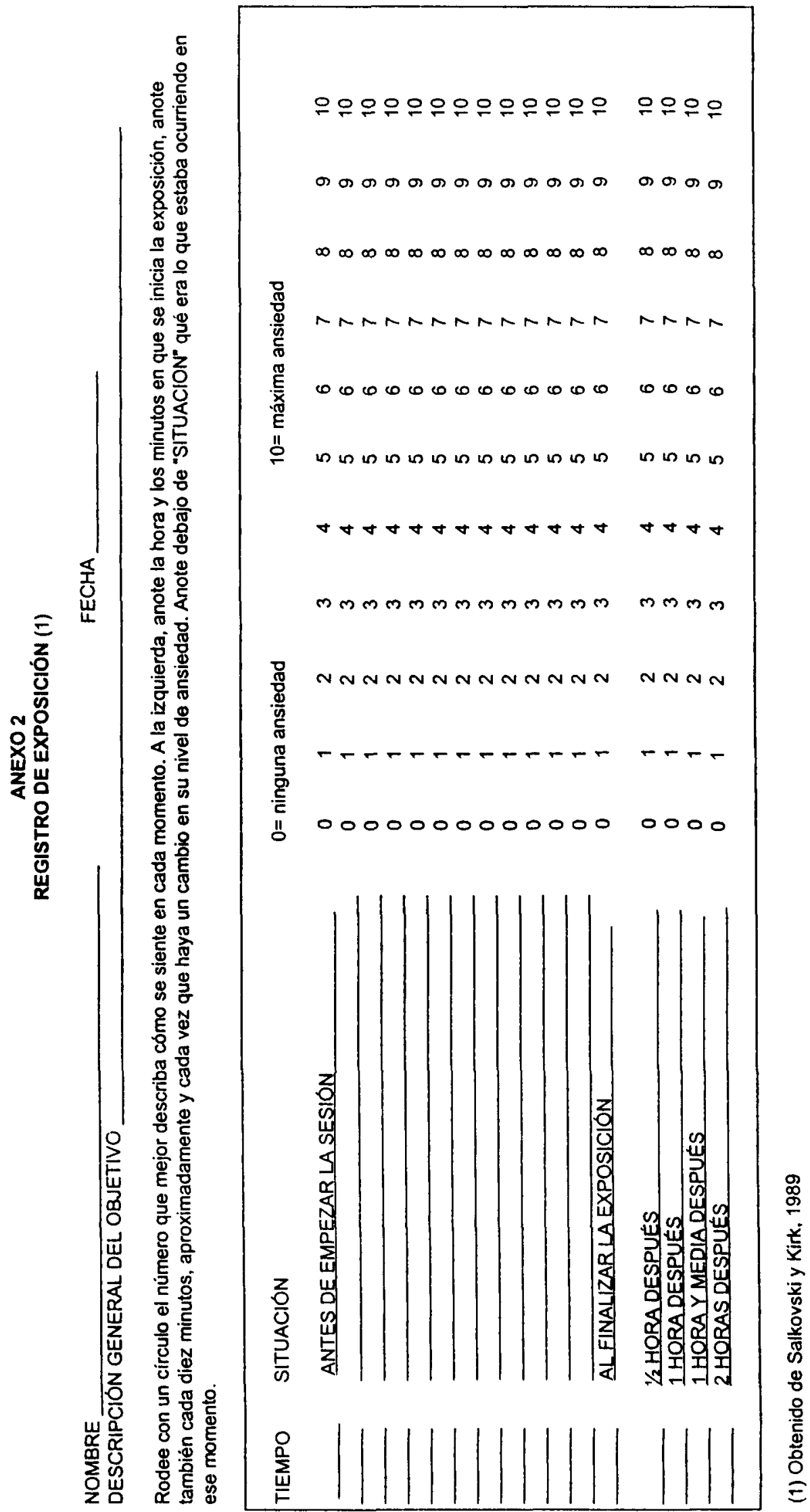




\section{ANEXO 3 \\ MANUAL DE EXPOSICIÓN PARA EL TRASTORNO OBSESIVO-COMPULSIVO (1)}

El trastorno obsesivo-compulsivo consiste en un miedo exagerado a que ocurra algo malo (por ejemplo: ser responsable de un accidente). Para impedir esto, la persona trata de evitar las situaciones que pudieran provocarlo (por ejemplo: coger el coche) y cuando esto no es posible, uno busca el medio de eliminar la posibilidad de que ocurra lo que uno teme, realizando determinadas acciones (por ejemplo: comprobando repetidas veces que no me he equivocado, que no le he dado a nadie).

Esto nos hace sentir bien a corto plazo, pues nos tranquiliza, pero a largo plazo está prolongando el problema, pues cada vez nos produce una mayor ansiedad la posibilidad de que algo haya ido mal, y cada vez nos ponemos más nerviosos si no podemos realizar la conducta tranquilizadora (comprobar).

El mejor tratamiento para este trastorno consiste en exponer a la persona a aquellas situaciones que le provocan ansiedad y/o está evitando, de modo contínuo y prolongado hasta que ésta disminuya.

Para ello Ud. tendrá que enfrentarse diariamente a las cosas que le producen miedo. La tarea debe durar el tiempo suficiente (una hora u hora y media) para permitir que disminuya su ansiedad. Es importante que reserve un tiempo para ello todos los días. Para empezar, anotará cuál es la meta de la sesión de hoy en la hoja de exposición del modo más concreto posible.

Comience por el punto más fácil de la lista que no puede llevar a cabo de momento. No debe abandonar una situación ni realizar ninguna conducta tranquilizadora hasta que la ansiedad haya disminuido.

Repita el mismo objetivo varias veces antes de pasar a uno más difícil. Después de practicado con éxito varias veces, introdúzcalo en su modo de vida habitual. Recuerde que la ansiedad es producida por la reacción de miedo (condicionada) a algunas situaciones. Estas reacciones se esfumarán con rapidez si se lo permite. Acepte estas sensaciones desagradables como lo que son. Recuerde que el afrontarlas sin huir ni rendirse forma parte de su recuperación.

- Sólo son sensaciones corporales desagradables que Ud. ya habia previsto. Si las acepta irán disminuyendo.

- No sucederá nada peor.

- Espere y deje tiempo al miedo para que pase. No luche ni huya de él.

- No añada pensamientos atemorizadores.

- Observe que cuando deja de añadir pensamientos atemorizadores, el miedo empieza a desaparecer.

- Recuerde que el objetivo de la práctica es aprender cómo afrontar el miedo. Es una oportunidad para progresar.

- Piense en el avance conseguido hasta ahora. Piense en lo satisfecho que estará cuando lo consiga esta vez.

- Anote la tarea que está practicando. Anote también su ansiedad cada vez que ésta experimenta un cambio, cada 10 minutos o al iniciar algo nuevo. Estas anotaciones le señalarán su progreso.

(1) Remodelado a partir de Mathews et al. (1981). 